\title{
On the edge-to-vertex geodetic number of a graph
}

\author{
A. P. Santhakumaran and J.. John
}




\title{
ON THE EDGE-TO-VERTEX GEODETIC NUMBER OF A GRAPH
}

\author{
A. P. SANTHAKUMARAN AND J. JOHN
}

Received 4 May, 2011

\begin{abstract}
Let $G=(V, E)$ be a connected graph with at least three vertices. For vertices $u$ and $v$ in $G$, the distance $d(u, v)$ is the length of a shortest $u-v$ path in $G$. A $u-v$ path of length $d(u, v)$ is called a $u-v$ geodesic. For subsets $A$ and $B$ of $V$, the distance $d(A, B)$, is defined as $d(A, B)=\min \{d(x, y): x \in A, y \in B\}$. A $u-v$ path of length $d(A, B)$ is called an $A-B$ geodesic joining the sets $A, B \subseteq V$, where $u \in A$ and $v \in B$. A vertex $x$ is said to lie on an $A-B$ geodesic if $x$ is a vertex of an $A-B$ geodesic. A set $S \subseteq E$ is called an edge-to-vertex geodetic set if every vertex of $G$ is either incident with an edge of $S$ or lies on a geodesic joining a pair of edges of $S$. The edge-to-vertex geodetic number $g_{e v}(G)$ of $G$ is the minimum cardinality of its edge-to-vertex geodetic sets and any edge-to-vertex geodetic set of cardinality $g_{e v}(G)$ is an edge-to-vertex geodetic basis of $G$. Any edge-to-vertex geodetic basis is also called a $g_{e v}$-set of $G$. It is shown that if $G$ is a connected graph of size $q$ and diameter $d$, then $g_{e v}(G) \leq q-d+2$. It is proved that, for a tree $T$ with $q \geq 2, g_{e v}(T)=q-d+2$ if and only if $T$ is a caterpillar. For positive integers $r, d$ and $l \geq 2$ with $r \leq d \leq 2 r$, there exists a connected graph $G$ with $r a d$ $G=r$, $\operatorname{diam} G=d$ and $g_{e v}(G)=l$. Also graphs $G$ for which $g_{e v}(G)=q, q-1$ or $q-2$ are characterized.
\end{abstract}

2000 Mathematics Subject Classification: $05 \mathrm{C} 12$

Keywords: distance, geodesic, edge-to-vertex geodetic basis, edge-to-vertex geodetic number

\section{INTRODUCTION}

By a graph $G=(V, E)$, we mean a finite undirected connected graph without loops or multiple edges. The order and size of $G$ are denoted by $p$ and $q$, respectively. We consider connected graphs with at least three vertices. For basic definitions and terminologies we refer to $[1,6]$. For vertices $u$ and $v$ in a connected graph $G$, the distance $d(u, v)$ is the length of a shortest $u-v$ path in $G$. An $u-v$ path of length $d(u, v)$ is called an $u-v$ geodesic. A vertex $v$ is said to lie on an $x-y$ geodesic $P$ if $v$ is a vertex of $P$ including the vertices $x$ and $y$. A vertex $v$ is an internal vertex of an $x-y$ path $P$ if $v$ is a vertex of $P$ and $v \neq x, y$. An edge $e$ of $G$ is an internal $e d g e$ of an $x-y$ path $P$ if $e$ is an edge of $P$ with both its ends internal vertices of $P$. An edge $e$ is a pendant edge if one of its ends is of degree 1. For a vertex $v$ of $G$, the eccentricity $e(v)$ is the distance between $v$ and a vertex farthest from $v$. The minimum eccentricity among the vertices of $G$ is the radius, $\mathrm{rad} G$ and the 
maximum eccentricity is its diameter, diam $G$ of $G$. A double star is a tree of diameter 3. The neighborhood of a vertex $v$ is the set $N(v)$ consisting of all vertices $u$ which are adjacent with $v$. A vertex $v$ is an extreme vertex of $G$ if the subgraph induced by its neighbors is complete.

The closed interval $I[x, y]$ consists of all vertices lying on some $x-y$ geodesic of $G$, while for $S \subseteq V, I[S]=\bigcup_{x, y \in S} I[x, y]$. A set $S$ of vertices is a geodetic set if $I[S]=V$, and the minimum cardinality of a geodetic set is the geodetic number $g(G)$. A geodetic set of cardinality $g(G)$ is called a $g$-set. The geodetic number of a graph was introduced in [7] and further studied in [2],[3] and [4]. It was shown in [7] that determining the geodetic number of a graph is an NP-hard problem. The forcing geodetic number of graph was introduced and studied in [5]. The connected geodetic number of graph was studied in [11]. The upper connected geodetic number and forcing connected geodetic number of a graph were studied in [12].

The edge geodetic number of a graph was studied by in [9]. An edge geodetic set of a connected graph $G$ with at least two vertices is a set $S \subseteq V$ such that every edge of $G$ is contained in a geodesic joining some pair of vertices in $S$. The edge geodetic number $g_{1}(G)$ of $G$ is the minimum order of its edge geodetic sets and any edge geodetic set of order $g_{1}(G)$ is an edge geodetic basis of $G$.

Consider the graph $G$ given in Figure 1. The sets $S=\left\{v_{3}, v_{5}\right\}$ and $S_{1}=\left\{v_{1}, v_{2}, v_{4}\right\}$ are minimum geodetic set and minimum edge geodetic set of $G$ respectively so that $g(G)=2$ and $g_{1}(G)=3$. These concepts have many applications in location the-

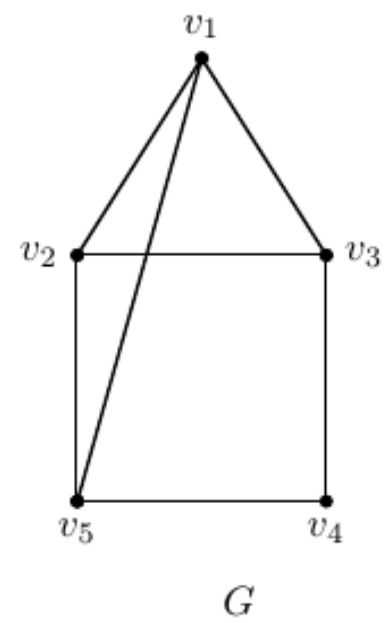

FIGURE 1.

ory and convexity theory. There are interesting applications of these concepts to the problem of designing the route for a shuttle and communication network design. We 
further extend these concepts to the edge set of $G$ and present several interesting results in [10].

Throughout the following $G$ denotes a connected graph with at least three vertices.

For subsets $A$ and $B$ of $V$, the distance $d(A, B)$ is defined as $d(A, B)=\min$ $\{d(x, y): x \in A, y \in B\}$. A $u-v$ path of length $d(A, B)$ is called an $A-B$ geodesic joining the sets $A, B$, where $u \in A$ and $v \in B$. A vertex $x$ is said to lie on an $A-B$ geodesic if $x$ is a vertex of an $A-B$ geodesic. For $A=\{u, v\}$ and $B=\{z, w\}$ with $u v$ and $z w$ edges, we write an $A-B$ geodesic as $u v-z w$ geodesic and $d(A, B)$ as $d(u v, z w)$.

For the graph $G$ given in Figure 2 with $A=\left\{v_{4}, v_{5}\right\}$ and $B=\left\{v_{1}, v_{2}, v_{7}\right\}$, the paths $P: v_{5}, v_{6}, v_{7}$ and $Q: v_{4}, v_{3}, v_{2}$ are the only two $A-B$ geodesics so that $d(A, B)=2$. A set $S \subseteq E$ is called an edge-to-vertex geodetic set if every vertex of $G$ is either

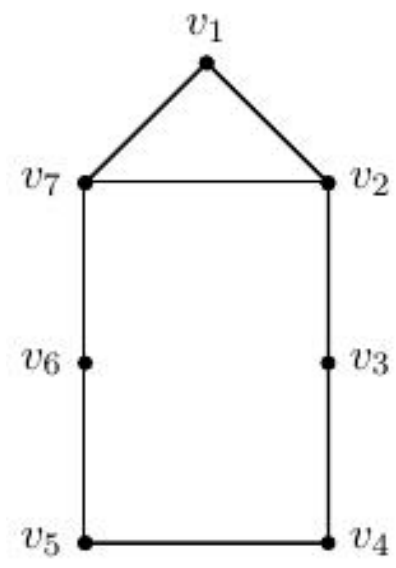

G

FIGURE 2.

incident with an edge of $S$ or lies on a geodesic joining a pair of edges of $S$. The edge-to-vertex geodetic number $g_{e v}(G)$ of $G$ is the minimum cardinality of its edgeto-vertex geodetic sets and any edge-to-vertex geodetic set of cardinality $g_{e v}(G)$ is an edge-to-vertex geodetic basis of $G$.

For the graph $G$ given in Figure 3, the three $v_{1} v_{6}-v_{3} v_{4}$ geodesics are $P: v_{1}, v_{2}, v_{3}$; $Q: v_{1}, v_{2}, v_{4}$; and $R: v_{6}, v_{5}, v_{4}$ with each of length 2 so that $d\left(v_{1} v_{6}, v_{3} v_{4}\right)=2$. Since the vertices $v_{2}$ and $v_{5}$ lie on the $v_{1} v_{6}-v_{3} v_{4}$ geodesics $P$ and $R$ respectively, $S=\left\{v_{1} v_{6}, v_{3} v_{4}\right\}$ is an edge-to-vertex geodetic basis of $G$ so that $g_{e v}(G)=2$. For the graph $G$ given in Figure 2, $S_{1}=\left\{v_{1} v_{2}, v_{1} v_{7}, v_{4} v_{5}\right\}$ and $S_{2}=\left\{v_{1} v_{2}, v_{4} v_{5}, v_{6} v_{7}\right\}$ are two $g_{e v}$-sets of $G$. Thus there can be more than one $g_{e v}$-set of $G$. 


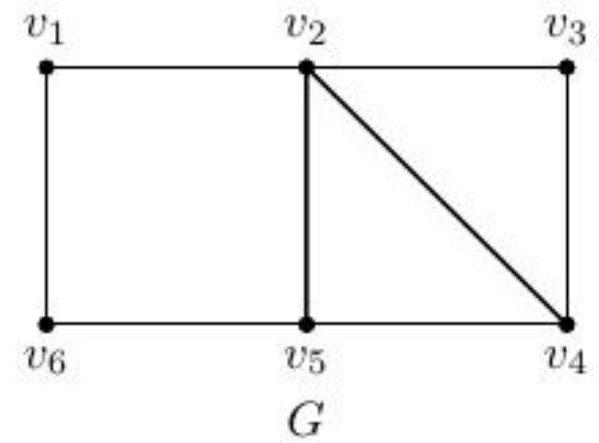

FIGURE 3 .

For a connected graph $G$ of size $q \geq 2$, it is clear that $2 \leq g_{e v}(G) \leq q$. Further, these bounds for $g_{e v}(G)$ are sharp. For the star $G=K_{1, q}(q \geq 2)$, it is clear that the set of all edges is the unique edge-to-vertex geodetic set so that $g_{e v}(G)=q$. The set of two end-edges of a path $P$ of length at least 2 is its unique edge-to-vertex geodetic basis so that $g_{e v}(P)=2$. Thus the star $K_{1, q}$ has the largest possible edge-to-vertex geodetic number $q$ and the paths of length at least 2 have the smallest edge-to-vertex geodetic number 2 .

An edge of a connected graph $G$ is called an extreme edge of $G$ if one of its ends is an extreme vertex of $G$. An edge $e$ of a connected graph $G$ is an edge-to-vertex geodetic edge in $G$ if e belongs to every edge-to-vertex geodetic basis of $G$. If $G$ has a unique edge-to-vertex geodetic basis $S$, then every edge in $S$ is an edge-to-vertex geodetic edge of $G$.

For the graph $G$ given in Figure $4, S=\{u x, z v\}$ is the unique edge-to-vertex geodetic basis so that both the edges in $S$ are edge-to-vertex geodetic edges of $G$. For the graph $G$ given in Figure 5, $S_{1}=\left\{v_{1} v_{2}, v_{6} v_{7}, v_{7} v_{8}\right\}, S_{2}=\left\{v_{1} v_{2}, v_{5} v_{6}, v_{7} v_{8}\right\}$ and $S_{3}=\left\{v_{1} v_{2}, v_{5} v_{8}, v_{6} v_{7}\right\}$ are the only $g_{e v}$-sets of $G$ so that every $g_{e v}$-set contains the edge $v_{1} v_{2}$. Hence the edge $v_{1} v_{2}$ is the unique edge-to-vertex geodetic edge of $G$. The following theorems from [10] are used in the sequel.

Theorem 1. If $v$ is an extreme vertex of a connected graph $G$, then every edge-tovertex geodetic set contains at least one extreme edge that is incident with $v$.

Theorem 2. Every pendant edge of a connected graph $G$ belongs to every edgeto-vertex geodetic set of $G$.

Theorem 3. For a non-trivial tree $T$ with $k$ end-vertices, $g_{e v}(T)=k$ and the set of all pendant edges of $T$ is the unique edge-to-vertex geodetic basis of $T$.

Theorem 4. For the complete graph $K_{p}(p \geq 4)$ with $p$ even, $g_{e v}\left(K_{p}\right)=p / 2$.

Theorem 5. For the cycle $C_{p}(p \geq 4), g_{e v}\left(C_{p}\right)= \begin{cases}2 & \text { if } p \text { is even } \\ 3 & \text { if } p \text { is odd }\end{cases}$ 


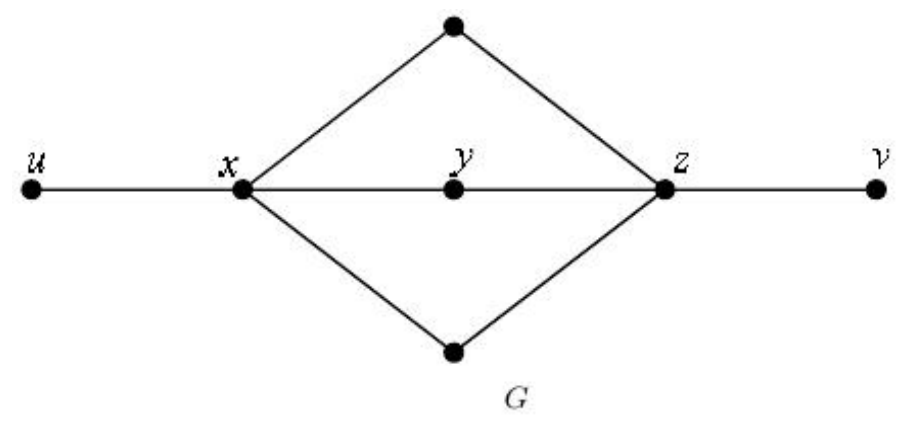

FIGURE 4.

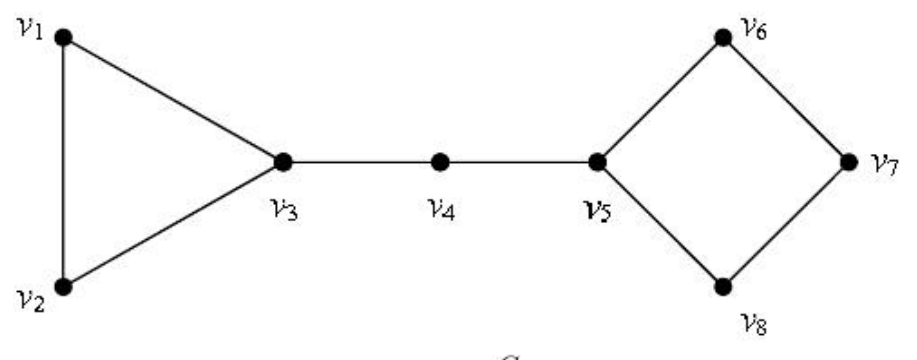

G

FIGURE 5 .

\section{THE EDGE-TO-VERTEX GEODETIC NUMBER AND DIAMETER OF A GRAPH}

If $G$ is a connected graph of size $q \geq 2$, then $2 \leq g_{e v}(G) \leq q$. An improved upper bound for the edge-to-vertex geodetic number of a graph can be given in terms of its size $q$ and diameter $d$.

Theorem 6. For a connected graph $G$ with $q \geq 2, g_{\text {ev }}(G) \leq q-d+2$, where $d$ is the diameter of $G$.

Proof. Let $u$ and $v$ be vertices of $G$ for which $d(u, v)=d$, where $d$ is the diameter of $G$ and let $P: u=v_{0}, v_{1}, v_{2}, \ldots, v_{d}=v$ be a $u-v$ path of length $d$. Let $e_{i}=$ $v_{i-1} v_{i}(1 \leq i \leq d)$. Let $S=E(G)-\left\{v_{1} v_{2}, v_{2} v_{3}, \ldots, v_{d-2} v_{d-1}\right\}$. Let $x$ be a vertex of $G$. If $x=v_{i}(1 \leq i \leq d-1)$, then $x$ lies on the $e_{1}-e_{d}$ geodesic $P_{1}: v_{1}, v_{2}, \ldots, v_{d-1}$. If $x \neq v_{i}(1 \leq i \leq d-1)$, then $x$ is incident with an edge of $S$. Therefore, $S$ is an edge-to-vertex geodetic set of $G$. Consequently, $g_{e v}(G) \leq|S|=q-d+2$.

Remark 1. The bound in Theorem 6 is sharp. For the $\operatorname{star} G=K_{1, q}(q \geq 2), d=2$ and $g_{e v}(G)=q$, by Theorem 3 so that $g_{e v}(G)=q-d+2$.

We give below a characterization theorem for trees.

A caterpillar is a tree for which the removal of all end-vertices leaves a path. 
Theorem 7. Let $q \geq 2$. For any tree $T$ with diameter $d, g_{e v}(T)=q-d+2$ if and only if $T$ is a caterpillar.

Proof. Let $P: v_{0}, v_{1}, \ldots, v_{d-1}, v_{d}$ be a diametral path of length $d$. Let $e_{i}=$ $v_{i-1} v_{i}(1 \leq i \leq d)$ be the edges of the diametral path $P$. Let $k$ be the number of pendant edges of $T$ and $l$ be the number of internal edges of $T$ other than $e_{i}(2 \leq$ $i \leq d-1)$. Then $d-2+l+k=q$. By Theorem $3, g_{e v}(T)=k$ and so $g_{e v}(T)=$ $q-d+2-l$. Hence $g_{e v}(T)=q-d+2$ if and only if $l=0$, if and only if all internal vertices of $T$ lie on the diametral path $P$, if and only if $T$ is a caterpillar.

The following theorem gives a realization result.

Theorem 8. For each triple $d, k, q$ of integers with $2 \leq k \leq q-d+2, d \geq 4$ and $q-d+k+1>0$, there exists a connected graph $G$ of size $q$ with diam $G=d$ and $g_{\text {ev }}(G)=k$.

Proof. Let $2 \leq k=q-d+2$. Let $G$ be the graph obtained from the path $P$ of length $d$ by adding $q-d$ new vertices to $P$ and joining them to a cut-vertex of $P$. Then $G$ is a tree of size $q$ and diam $G=d$. By Theorem $3, g_{e v}(G)=q-d+2=k$. Now, let $2 \leq k<q-d+2$.

Case 1. $q-d-k+1$ is even. Let $(q-d-k+1) \geq 2$. Let $n=\frac{q-d-k+1}{2}$. Then $n \geq 1$. Let $P_{d}: u_{0}, u_{1}, \ldots, u_{d}$ be a path of length $d$. Add new vertices $v_{1}, v_{2}, \ldots, v_{k-2}$ and $w_{1}, w_{2}, \ldots, w_{n}$ and join each $v_{i}(1 \leq i \leq k-2)$ with $u_{1}$ and also join each $w_{i}(1 \leq i \leq n)$ with $u_{1}$ and $u_{3}$ in $P_{d}$. Now, join $w_{1}$ with $u_{2}$ and we obtain the graph $G$ in Figure 6 . Then $G$ has size $q$ and diameter $d$. By Theorem 2, all the pendant edges $u_{1} v_{i}(1 \leq i \leq k-2), u_{0} u_{1}$ and $u_{d-1} u_{d}$ lie in every edge-to-vertex geodetic set of $G$. Let $S=\left\{u_{1} v_{1}, u_{1} v_{2}, \ldots, u_{1} v_{k-2}, u_{1} u_{0}, u_{d-1} u_{d}\right\}$ be the set of all pendant edges of $G$. Then it is clear that $S$ is an edge-to-vertex geodetic set of $G$ and so $g_{e v}(G)=k$.

Case 2. $q-d-k+1$ is odd. Let $q-d-k+1 \geq 5$. Let $m=\frac{q-d-k}{2}$. Then $m \geq 2$. Let $P_{d}: u_{0}, u_{1}, \ldots, u_{d}$ be a path of length $d$. Add new vertices $v_{1}, v_{2}, \ldots, v_{k-2}$ and $w_{1}, w_{2}, \ldots, w_{m}$ and join each $v_{i}(1 \leq i \leq k-2)$ with $u_{1}$ and also join each $w_{i}(1 \leq$ $i \leq m)$ with $u_{1}$ and $u_{3}$ in $P_{d}$. Now join $w_{1}$ and $w_{2}$ with $u_{2}$ and we obtain the graph $G$ in Figure 7. Then $G$ has size $q$ and diameter $d$. Now, as in Case $1, S=$ $\left\{u_{1} v_{1}, u_{1} v_{2}, \ldots, u_{1} v_{k-2}, u_{0} u_{1}, u_{d-1} u_{d}\right\}$ is an edge-to-vertex geodetic set of $G$ so that $g_{e v}(G)=k$. Let $q-d-k+1=1$. Let $P_{d}: u_{0}, u_{1}, \ldots, u_{d}$ be a path of length $d$. Add new vertices $v_{1}, v_{2}, \ldots, v_{k-2}$ and $w_{1}$ and join each $v_{i}(1 \leq i \leq k-2)$ with $u_{1}$ and also join $w_{1}$ with $u_{1}$ and $u_{3}$ in $P_{d}$, there by obtaining the graph $G$ in Figure 8. Then the graph is of size $q$ and diameter $d$. Now, as in Case $1, S=$ $\left\{u_{1} v_{1}, u_{1} v_{2}, \ldots, u_{1} v_{k-2}, u_{0} u_{1}, u_{d-1} u_{d}\right\}$ is an edge-to-vertex geodetic set of $G$ so that $g_{e v}(G)=k$.

Now, let $q-d-k+1=3$. Let $P_{d}: u_{0}, u_{1}, \ldots, u_{d}$ be a path of length $d$. Add new vertices $v_{1}, v_{2}, v_{3}, \ldots, v_{k-2}, w_{1}$ and $w_{2}$ and join each $v_{i}(1 \leq i \leq k-2)$ with $u_{1}$ and 


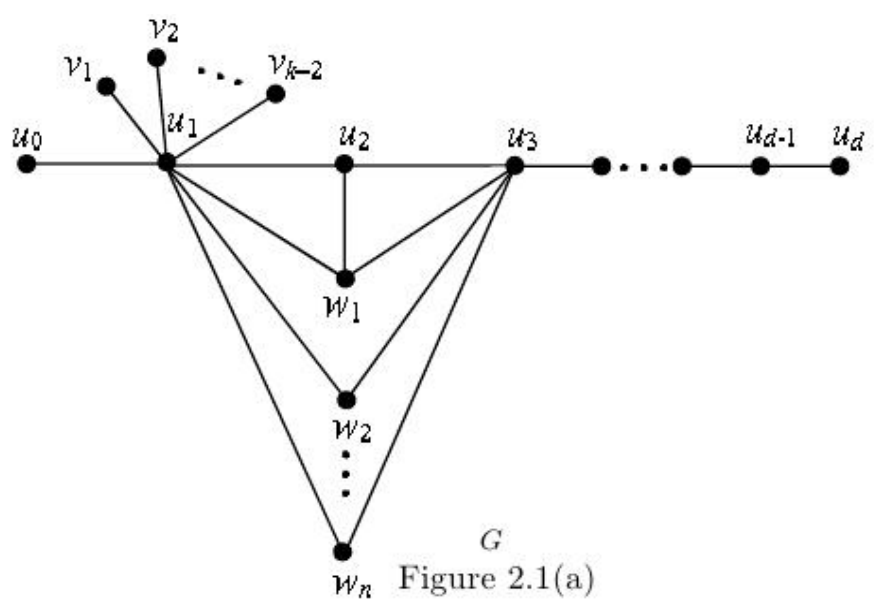

FIGURE 6.

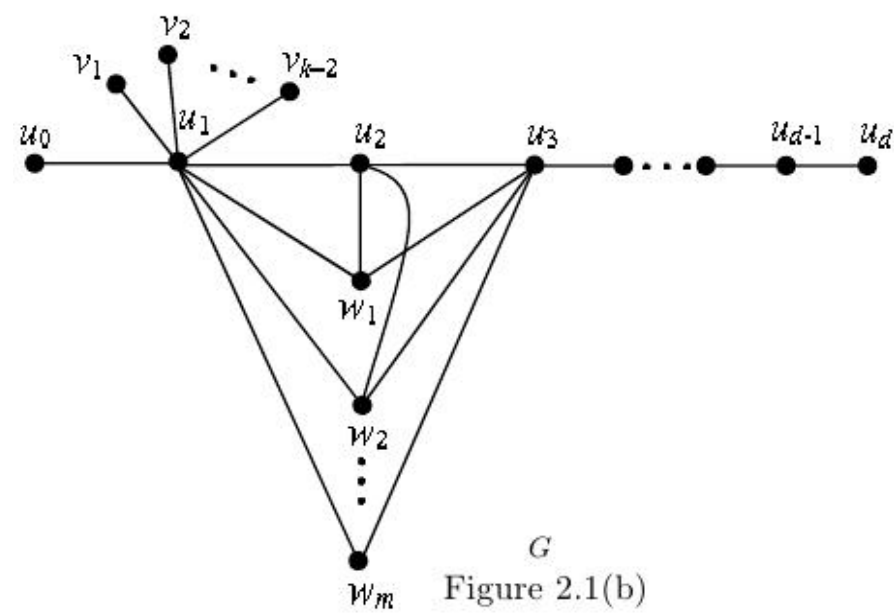

FIGURE 7.

also join $w_{1}$ and $w_{2}$ with $u_{1}$ and $u_{3}$ and obtain the graph $G$ in Figure 9. Then $G$ has size $q$ and diameter $d$. Now, as in Case $1, S=\left\{u_{1} v_{1}, u_{1} v_{2}, \ldots, u_{1} v_{k-2}, u_{0} u_{1}\right.$, $\left.u_{d-1} u_{d}\right\}$ is an edge-to-vertex geodetic set of $G$ so that $g_{e v}(G)=k$.

For every connected graph, rad $G \leq \operatorname{diam} G \leq 2 \mathrm{rad} G$. Ostrand [8] showed that every two positive integers $a$ and $b$ with $a \leq b \leq 2 a$ are realizable as the radius and diameter, respectively, of some connected graph. Now, Ostrand's theorem can be extended so that the edge-to-vertex geodetic number can also be prescribed. 


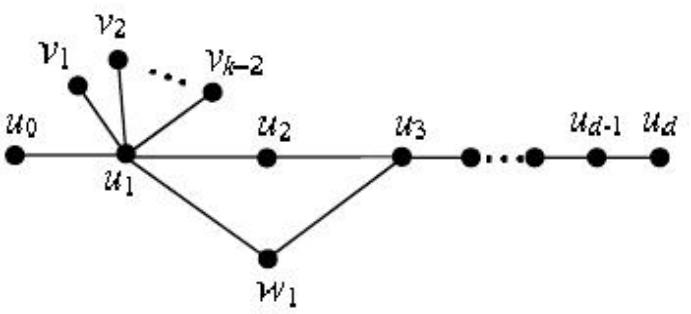

$G$

FIGURE 8.

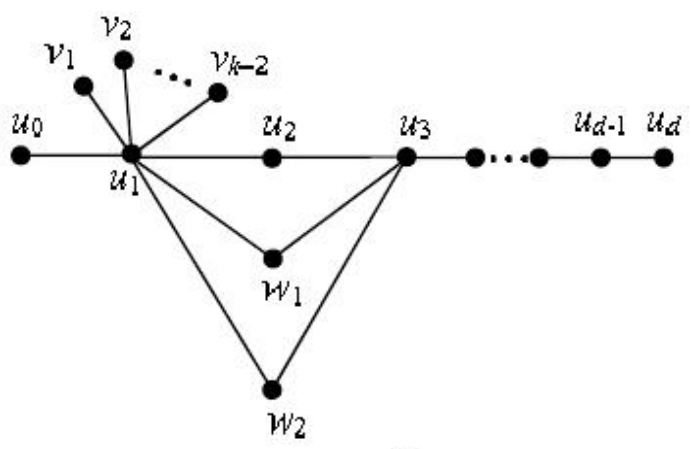

$G$

\section{FIGURE 9.}

Theorem 9. For positive integers $r, d$ and $l \geq 2$ with $r \leq d \leq 2 r$, there exists $a$ connected graph $G$ with rad $G=r$, diam $G=d$ and $g_{e v}(G)=l$.

Proof. When $r=1$, we let $G=K_{2 l}$ or $G=K_{1, l}$ according to whether $d=$ 1 or $d=2$ respectively. Then the result follows from Theorem 4 and Theorem 3 respectively. Let $r \geq 2$. If $r=d$ and $l=2$, let $G=C_{2 r}$. Then by Theorem 5, $g_{e v}(G)=2=l$. Let $l \geq 3$. Let $C_{2 r}: u_{1}, u_{2}, \ldots, u_{2 r}, u_{1}$ be the cycle of order $2 r$. Let $G$ be the graph obtained by adding the new vertices $x_{1}, x_{2}, \ldots, x_{l-1}$ and joining each $x_{i}(1 \leq i \leq l-1)$ with $u_{1}$ and $u_{2}$ of $C_{2 r}$. The graph $G$ is shown in Figure 10. It is easily verified that the eccentricity of each vertex of $G$ is $r$ so that $\operatorname{rad} G=\operatorname{diam}$ $G=r$. Let $S=\left\{u_{1} x_{1}, u_{1} x_{2}, \ldots, u_{1} x_{l-2}, u_{2} x_{l-1}\right\}$. It is clear that $S$ is not an edgeto-vertex geodetic set of $G$. However, $S \cup\left\{u_{r+1} u_{r+2}\right\}$ is an edge-to-vertex geodetic set of $G$. Since $x_{1}, x_{2}, \ldots, x_{l-1}$ are the only extreme vertices of $G$, it follows from Theorem 1 that $g_{e v}(G)=l$.

Let $r<d$. If $l=2$, then take $G$ to be any path on at least three vertices. Let $l \geq 3$. Let $C_{2 r}: v_{1}, v_{2}, \ldots, v_{2 r}, v_{1}$ be a cycle of order $2 r$ and let $P_{d-r+1}: u_{0}, u_{1}, u_{2}, \ldots$, $u_{d-r}$ be a path of order $d-r+1$. Let $H$ be the graph obtained from $C_{2 r}$ and $u_{0}$ in $P_{d-r+1}$ by identifying $v_{1}$ in $C_{2 r}$ and $u_{0}$ in $P_{d-r+1}$. Now, add $(l-3)$ new vertices 


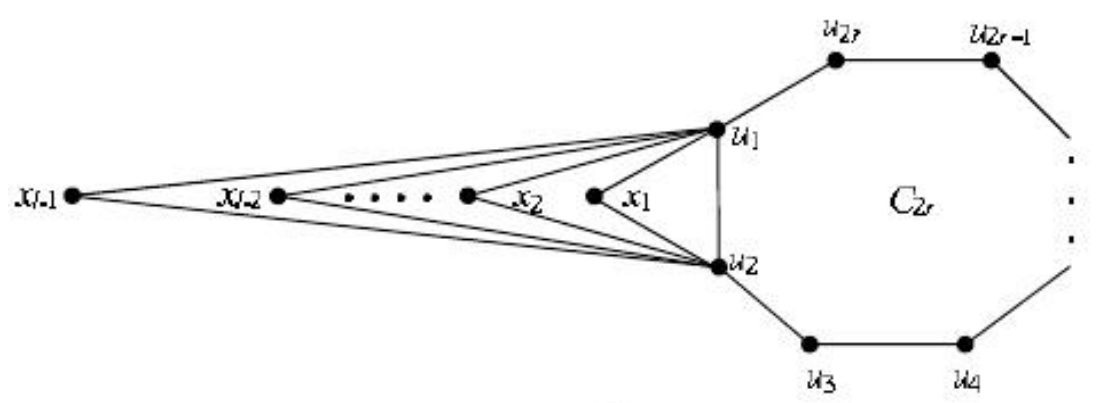

G

FIGURE 10.

$w_{1}, w_{2}, \ldots, w_{l-3}$ to $H$ and join each vertex $w_{i}(1 \leq i \leq l-3)$ to the vertex $u_{d-r-1}$ and obtain the graph $G$ of Figure 11. Then $\operatorname{rad} G=r$ and diam $G=d$. Let $S=$ $\left\{u_{d-r-1} w_{1}, u_{d-r-1} w_{2}, \ldots, u_{d-r-1} w_{l-3}, u_{d-r-1} u_{d-r}\right\}$ be the set of pendant edges of $G$. By Theorem 2, $S$ is contained in every edge-to-vertex geodetic set of $G$. It is clear that $S$ is not an edge-to-vertex geodetic set of $G$. It is also seen that $S \cup\{e\}$, where $e \in E(G)-S$ is not an edge-to-vertex geodetic set of $G$. However, the set $S_{1}=$ $S \cup\left\{v_{r} v_{r+1}, v_{r+1} v_{r+2}\right\}$ is an edge-to-vertex geodetic set of $G$ so that $g_{e v}(G)=$ $l-2+2=l$.

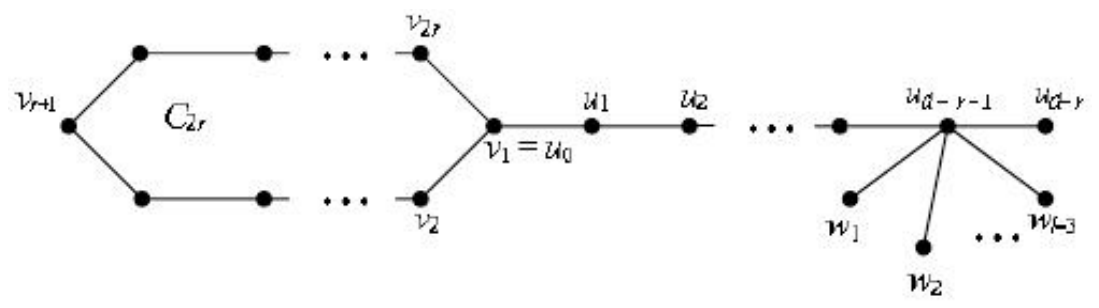

$G$

FIGURE 11.

\section{GRAPHS $G$ WITH $g_{e v}(G)=q, q-1$ AND $q-2$}

In the following we characterize graphs $G$ for which $g_{e v}(G)=q, q-1$ or $q-2$. Let $G$ be a graph. A subset $M \subseteq E(G)$ is called a matching of $G$ if no pair of edges in $M$ are incident. The maximum size of such $M$ is called the matching number of $G$ and is denoted by $\alpha^{\prime}(G)$. An edge covering of $G$ is subset $K \subseteq E(G)$ such that each vertex of $G$ is an end of some edge in $K$. The number of edges in a minimum edge covering of $G$, denoted by $\beta^{\prime}(G)$, is the edge covering number of $G$. The wellknown Gallai's theorem states that if $q \geq 1$, then $\alpha^{\prime}(G)+\beta^{\prime}(G)=p$. Since every 
edge covering for $G$ is an edge-to-vertex geodetic set, we have the following.

Lemma A. For any graph $G, g_{e v}(G) \leq \beta^{\prime}(G)=p-\alpha^{\prime}(G)$.

We will make use of this lemma in the sequel. The proofs of the next two theorems are straightforward.

Theorem 10. If $G$ is a connected graph such that it is not a star, then $g_{\text {ev }}(G) \leq$ $q-1$.

Theorem 11. For any connected graph $G, g_{e v}(G)=q$ if and only if $G$ is a star.

Theorem 12. Let $G$ be a connected graph which is not a tree. Then $g_{e v}(G) \leq q-2$ $(q \geq 4)$.

Proof. Since $G \neq C_{3}$ and it has atleast one cycle, $\alpha^{\prime}(G) \geq 2$. Thus, by Lemma A, $g_{\text {ev }}(G) \leq p-\alpha^{\prime}(G) \leq q-\alpha \prime(G) \leq q-2$.

Theorem 13. For any connected graph $G$ with $q \geq 3, g_{\text {ev }}(G)=q-1$ if and only if $G$ is either $C_{3}$ or a double star.

Proof. If $G$ is $C_{3}$, then $g_{e v}(G)=2=q-1$. If $G$ is a double star, then by Theorem $3, g_{e v}(G)=q-1$. Conversely, let $g_{e v}(G)=q-1$. If $G$ is a tree, then from Lemma A it follows that $\alpha^{\prime}(G) \leq 2$. If $\alpha^{\prime}(G)=1$, then $G$ is a star, which is impossible due to Theorem 11. So $\alpha^{\prime}(G)=2$, which implies that $G$ is a double star. If $G$ is not a tree, then $g_{e v}(G)=q-1 \geq p-1$. Again by Lemma A, $\alpha^{\prime}(G)=1$, which is the case only when $G=C_{3}$. Thus the proof is complete.

Theorem 14. Let $G$ be a connected graph with $q \geq 4$, which is not a cycle and not a tree and let $C(G)$ be the length of a smallest cycle. Then $g_{e v}(G) \leq q-C(G)+1$ if $C(G)$ is odd, and $g_{e v}(G) \leq q-C(G)+2$ if $C(G)$ is even.

Proof. Let $C(G)$ denote the length of a smallest cycle in $G$ and let $C$ be a cycle of length $C(G)$. We consider two cases.

Case 1. $C(G)$ is odd. First suppose that $C(G)=3$. Let $C: v_{1}, v_{2}, v_{3}, v_{1}$ be a cycle of length 3. Since $G$ is not a cycle, there exists a vertex $v$ in $G$ such that $v$ is not on $C$ and $v$ is adjacent to $v_{1}$, say. Let $S=E(G)-\left\{v_{1} v_{2}, v_{1} v_{3}\right\}$. Then every vertex of $G$ lies on an edge of $S$ and so $S$ is an edge-to-vertex geodetic set of $G$ set of $G$. Thus $g_{\text {ev }}(G) \leq q-2=q-C(G)+1$.

Next suppose that $C(G) \geq 5$. Let $C: v_{1}, v_{2}, \ldots, v_{k}, v_{k+1}, v_{k+2}, \ldots, v_{2 k+1}, v_{1}$ be a cycle of least length $C(G)=2 k+1$. Since $G$ is not a cycle, there exists a vertex $v$ in $G$ such that $v$ is not on $C$ and $v$ is adjacent to $v_{1}$, say. We claim that $d\left(v v_{1}, v_{k+1} v_{k+2}\right)=k$. Since $P: v_{1}, v_{2}, v_{3}, \ldots, v_{k+1}$ is a path of length $k$ on $C$, it follows that $d\left(v v_{1}, v_{k+1} v_{k+2}\right) \leq k$. If $d\left(v v_{1}, v_{k+1} v_{k+2}\right) \leq k-1$, then at least one 
of $d\left(v_{1}, v_{i}\right)$ and $d\left(v, v_{i}\right)$ for $i=k+1, k+2$ is less than or equal to $k-1$. First suppose that $d\left(v_{1}, v_{k+1}\right) \leq k-1$. Let $Q$ be a $v_{1}-v_{k+1}$ shortest path of length at most $k-1$ different from $P$. Hence there exists at least one vertex of $Q$ that is not on $P$ and since the length of $Q$ is at most $k-1$, it follows that a cycle of length at most $2 k-1$ is formed. This is a contradiction to $C(G)=2 k+1$. Thus $d\left(v_{1}, v_{k+1}\right)=k$. Similarly we can prove that $d\left(v_{1}, v_{k+2}\right)=k$.

Next, suppose that $d\left(v, v_{k+1}\right) \leq k-1$. Since $P^{\prime}: v, v_{1}, v_{2}, v_{3}, \ldots, v_{k+1}$ is a path of length $k+1$, it follows that $d\left(v, v_{k+1}\right) \leq k+1$. Then, as above, a cycle of length at most $2 k$ is formed and this is a contradiction. Hence $d\left(v, v_{k+1}\right)=k$ or $k+1$. Similarly we can prove that $d\left(v, v_{k+2}\right)=k$ or $k+1$. Since $d\left(v_{1}, v_{k+1}\right)=d\left(v_{1}, v_{k+2}\right)=$ $k$, it follows that $d\left(v v_{1}, v_{k+1} v_{k+2}\right)=k$.

Now, let $S=(E(G)-E(C)) \bigcup\left\{v_{k+1} v_{k+2}\right\}$. It is clear that the vertices $v_{2}, v_{3}, \ldots$, $v_{k}, v_{k+3}, v_{k+4}, \ldots, v_{2 k+1}$ on the cycle $C$ lie on the $v v_{1}-v_{k+1} v_{k+2}$ geodesic on the cycle and all the other vertices of $G$ are incident with an edge of $S$. Thus $S$ is an edgeto-vertex geodetic set of $G$ and so $g_{e v}(G) \leq q-C(G)+1$.

Case 2. $C(G)$ is even. First suppose that $C(G)=4$. Let $C: v_{1}, v_{2}, v_{3}, v_{4}, v_{1}$ be a cycle of length 4 . Since $G$ is not a cycle, there exists a vertex $v$ in $G$ such that $v$ is not on $C$ and $v$ is adjacent to $v_{1}$, say. Let $S=E(G)-\left\{v_{1} v_{2}, v_{1} v_{4}\right\}$. Then every vertex of $G$ lies on an edge of $S$ and so $S$ is an edge-to-vertex geodetic set of $G$. Thus $g_{e v}(G) \leq q-2=q-C(G)+2$.

Next suppose that $C(G) \geq 6$. Let $C: v_{1}, v_{2}, \ldots . v_{k}, v_{k+1}, v_{k+2}, \ldots, v_{2 k}, v_{1}$ be a cycle of least length $C(G)=2 k$. Since $G$ is not a cycle, there exists a vertex $v$ in $G$ such that $v$ is not on $C$ and $v$ is adjacent to $v_{1}$, say. We claim that $d\left(v v_{1}, v_{k} v_{k+1}\right)=$ $d\left(v v_{1}, v_{k+1} v_{k+2}\right)=k-1$. Since $Q: v_{1}, v_{2}, v_{3}, \ldots, v_{k}$ and $Q^{\prime}: v_{1}, v_{2 k}, v_{2 k-1}, \ldots$, $v_{k+3}, v_{k+2}$ are paths of length $k-1$ on $C$, it follows that $d\left(v v_{1}, v_{k} v_{k+1}\right)=d\left(v v_{1}, v_{k+1} v_{k+2}\right) \leq k-1$. If $d\left(v v_{1}, v_{k} v_{k+1}\right) \leq k-2$ or $d\left(v v_{1}, v_{k+1} v_{k+2}\right) \leq k-2$, then proceeding as in Case 1, a cycle of length at most $2 k-3$ or $2 k-2$ or $2 k-1$ is formed as the case may be, contradicting that the least length of a cycle is $2 k$. Thus $d\left(v v_{1}, v_{k} v_{k+1}\right)=d\left(v v_{1}, v_{k+1} v_{k+2}\right)=k-1$.

Now, if we let $S=(E(G)-E(C)) \bigcup\left\{v_{k} v_{k+1}, v_{k+1} v_{k+2}\right\}$, then the vertices $v_{2}, v_{3}, \ldots, v_{k-1}$ lie on the $v v_{1}-v_{k} v_{k+1}$ geodesic on $C$, the vertices $v_{k+3}, v_{k+4}, \ldots$, $v_{2 k}$ lie on the $v v_{1}-v_{k+1} v_{k+2}$ geodesic on $C$ and all the other vertices of $G$ are incident with an edge of $S$. Thus $S$ is an edge-to-vertex geodetic set of $G$ and so $g_{e v}(G) \leq q-C(G)+2$.

Theorem 15. If $G$ is a connected graph of size $q \geq 4$ and not a tree such that $g_{\text {ev }}(G)=q-2$, then $G$ is unicyclic.

Proof. Let $G$ have more than one cycle. Then $q \geq p+1$ and so $p-1 \leq q-2=$ $g_{e v}(G) \leq p-\alpha^{\prime}(G)$, by Lemma A. Hence $\alpha^{\prime}(G)=1$ and so $G$ must be either a star or the cycle $C_{3}$, a contradiction. 
Denote by $\mathfrak{s}$ the two classes of graphs given in Figure 12.

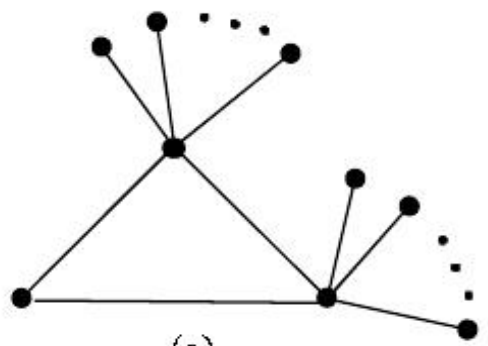

(a)

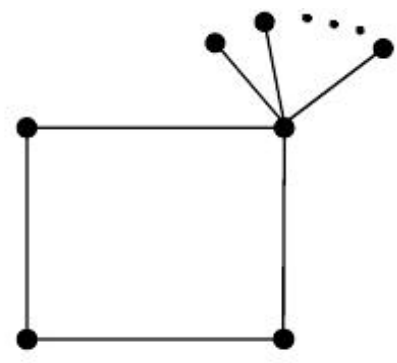

(b)

FIGURE 12.

Theorem 16. For a connected graph $G, g_{\text {ev }}(G)=q-2(q \geq 4)$ if and only if $G$ is $C_{4}$ or $C_{5}$ or $K_{1, q-1}+e$ or caterpillar with $d=4$ or the class of graphs given in family $\mathfrak{s}$ of Figure 12.

Proof. For $G=C_{4}$ or $C_{5}$, the result follows from Theorem 5. For a caterpillar of diameter 4, the result follows from Theorem 3. For $G=K_{1, q-1}+e$, it follows from Theorem 1 that the set of all end edges of $G$ together with $e$ forms an edge-to-vertex geodetic basis so that $g_{e v}(G)=q-2$. Further, it is easily verified that $g_{e v}(G)=q-2$ for the graphs given in family $\mathfrak{s}$ of Figure 12.

Now, let $G$ be a connected graph such that $g_{e v}(G)=q-2$. By Theorem $15, G$ is either a tree or unicyclic. If $G$ is a tree, then from Lemma A it follows that $\alpha^{\prime}(G) \leq 3$. By Theorems 12 and 13, $\alpha^{\prime}>2$. So $\alpha^{\prime}=3$, which implies that $G$ is a Caterpillar of diameter 4. If $G$ is unicyclic, by Lemma A, $\alpha^{\prime}(G) \leq 2$. Let $C_{k}$ be the unique cycle of $G$. We have $k \leq 5$ since otherwise $\alpha^{\prime}(G) \geq \alpha^{\prime}\left(C_{k}\right) \geq 3$. Therefore, we have the following three cases:

Case 1. $k=5$. Then $G$ cannot have any other vertices since otherwise $\alpha^{\prime}(G) \geq 3$. Therefore $G=C_{5}$.

Case 2. $k=4$. If $G=C_{4}$, we are done. So, let $G \neq C_{4}$. Because $\alpha^{\prime}(G) \leq 2$, only one of the vertices of $C_{4}$, say $v$, is of degree more than 2 and moreover all the neighbors of $v$ are of degree 1 . Thus $G$ should be a graph like Figure 12(b).

Case 3. $k=3$. Since $g_{\text {ev }}\left(C_{3}\right)=2=q-1$, we have $G \neq C_{3}$. Let $V\left(C_{3}\right)=$ $\left\{v_{1}, v_{2}, v_{3}\right\}$. We note that if $u \in V(G)-V\left(C_{3}\right)$, then $\operatorname{deg} u=1$. Otherwise, there are $u_{1}, u_{2} \in V(G)-V\left(C_{3}\right)$ such that $u_{1}$ is adjacent to both $u_{2}$ and $v_{1}$, say. Then it is easily seen that $E(G)-\left\{u_{1} v_{1}, v_{1} v_{2}, v_{1} v_{3}\right\}$ is a edge-to-vertex geodetic set, which implies $g_{e v}(G) \leq q-3$. Further, at least one of the $v_{i}$ s should be of degree 2. Otherwise, $E(G)-E\left(C_{3}\right)$ is a edge-to-vertex geodetic set, which is impossible. Thus $G$ should be either $K_{1, q}+e$ or a graph like Figure 12(a). 


\section{ACKNOWLEDGEMENT}

The authors express their gratitude to the referees for the useful suggestions.

\section{REFERENCES}

[1] F. Buckley and F. Harary, Distance in graphs. Redwood City: Addison-Wesley, 1990.

[2] F. Buckley, F. Harary, and L. V. Quintas, "Extremal results on the geodetic number of a graph," Sci., Ser. A, vol. 2, pp. 17-26, 1988.

[3] G. Chartrand, F. Harary, and P. Zhang, "On the geodetic number of a graph," Networks, vol. 39, no. 1, pp. 1-6, 2002.

[4] G. Chartrand, E. M. Palmer, and P. Zhang, "The geodetic number of a graph: A survey," Congr. Numerantium, vol. 156, pp. 37-58, 2002.

[5] G. Chartrand and P. Zhang, "The forcing geodetic number of a graph," Discuss. Math., Graph Theory, vol. 19, no. 1, pp. 45-58, 1999.

[6] F. Harary, Graph theory, ser. Addison-Wesley Series in Mathematics. Reading, Mass.: AddisonWesley Publishing Company, 1969, vol. IX.

[7] F. Harary, E. Loukakis, and C. Tsouros, "The geodetic number of a graph," Math. Comput. Modelling, vol. 17, no. 11, pp. 89-95, 1993.

[8] P. A. Ostrand, "Graphs with specified radius and diameter," Discrete Math., vol. 4, pp. 71-75, 1973.

[9] A. P. Santhakumaran and J. John, "Edge geodetic number of a graph," J. Discrete Math. Sci. Cryptography, vol. 10, no. 3, pp. 415-432, 2007.

[10] A. P. Santhakumaran and J. John, "The edge-to vertex geodetic number of a graph," communicated.

[11] A. P. Santhakumaran, P. Titus, and J. John, "On the connected geodetic number of a graph," $J$. Comb. Math. Comb. Comput., vol. 69, pp. 219-229, 2009.

[12] A. P. Santhakumaran, P. Titus, and J. John, "The upper connected geodetic number and forcing connected geodetic number of a graph,” Discrete Appl. Math., vol. 157, no. 7, pp. 1571-1580, 2009.

Authors' addresses

A. P. Santhakumaran

Department of Mathematics, St. Xavier's College (Autonomous), Palayamkottai - 627 002, India.

E-mail address: apskumar19530yahoo.co.in

\section{J. John} India.

Department of Mathematics, A.C. College of Engineering and Technology, Karaikudi - 630004 , 